\title{
Impact of energy deficit calculated by a predictive method on outcome in medical patients requiring prolonged acute mechanical ventilation
}

\author{
Christophe Faisy ${ }^{1,2 *}$, Nicolas Lerolle ${ }^{1,2}$, Fahmi Dachraoui ${ }^{1,2}$, Jean-François Savard ${ }^{1,2,3}$ Imad Abboud $^{1,2}$, \\ Jean-Marc Tadie ${ }^{1,2}$ and Jean-Yves Fagon ${ }^{1,2}$ \\ ${ }^{1}$ Assistance Publique-Hôpitaux de Paris, Hôpital Européen Georges Pompidou, Service de Réanimation Médicale, 20 rue Leblanc, \\ 75908, Paris, France \\ ${ }^{2}$ Université Paris-Descartes, Paris, France \\ ${ }^{3}$ Intensive Care Unit, Hôpital de l'Enfant-Jésus 1401 18ème rue Québec, Québec G1J 1Z4, Canada
}

(Received 8 April 2008 - Revised 5 June 2008 - Accepted 30 June 2008 - First published online 9 September 2008)

To assess energy balance in very sick medical patients requiring prolonged acute mechanical ventilation and its possible impact on outcome, we conducted an observational study of the first $14 \mathrm{~d}$ of intensive care unit (ICU) stay in thirty-eight consecutive adult patients intubated at least $7 \mathrm{~d}$ Exclusive enteral nutrition (EN) was started within $24 \mathrm{~h}$ of ICU admission and progressively increased, in absence of gastrointestinal intolerance, to the recommended energy of $125.5 \mathrm{~kJ} / \mathrm{kg}$ per d. Calculated energy balance was defined as energy delivered - resting energy expenditure estimated by a predictive method based on static and dynamic biometric parameters. Mean energy balance was -5439 (SEM 222 ) $\mathrm{kJ}$ per d. EN was interrupted $23 \%$ of the time and situations limiting feeding administration reached $64 \%$ of survey time. ICU mortality was $72 \%$. Non-survivors had higher mean energy deficit than ICU survivors $(P=0.004)$. Multivariate analysis identified mean energy deficit as independently associated with ICU death $(P=0 \cdot 02)$. Higher ICU mortality was observed with higher energy deficit $(P=0 \cdot 003$ comparing quartiles). Using receiver operating characteristic curve analysis, the best deficit threshold for predicting ICU mortality was $5021 \mathrm{~kJ}$ per d. Kaplan-Meier analysis showed that patients with mean energy deficit $\geqq 5021 \mathrm{~kJ}$ per $\mathrm{d}$ had a higher ICU mortality rate than patients with lower mean energy deficit after the 14th ICU day $(P=0.01)$. The study suggests that large negative energy balance seems to be an independent determinant of ICU mortality in a very sick medical population requiring prolonged acute mechanical ventilation, especially when energy deficit exceeds $5021 \mathrm{~kJ}$ per $\mathrm{d}$.

Energy balance: Enteral nutrition: Acute prolonged mechanical ventilation: Outcome

Nutritional support is based on the assumption that critically ill patients are prone to develop malnutrition, especially protein-energy deficit, this condition being associated with morbidity and mortality ${ }^{(1-5)}$. Indeed, protein-energy deficit seems common in intensive care units (ICU), occurring in $43-88 \%$ of critically ill patients ${ }^{(6,7)}$. Underfeeding has been reported as associated with an increased rate of infection, poor wound healing, reduced respiratory muscle mass, delayed weaning from mechanical ventilation, increased length of ICU stay and increased health care costs ${ }^{(1,8-13)}$.

Perturbations of the normal metabolic response to starvation with hyperglycaemia, high lactate level, hypertriglyceridaemia and high NEFA concentrations due to insulin resistance characterize the hypermetabolic state of the critically injured patients ${ }^{(2,14,15)}$. Energy deficit results from a combination of hypermetabolism and reduced intake due to frequent interruptions in feedings because of gastrointestinal intolerance, diagnostic and therapeutic procedures ${ }^{(16-18)}$. In intubated and mechanically ventilated patients, the great variability of resting energy expenditure (REE) and nutrient delivery compared to prescription, partly due to frequent use of sedatives, analgesics or vasoconstrictors, increases the risk of mismatch between energy requirements and intakes ${ }^{(16,19-21)}$.

Enteral nutrition (EN) became widespread in ICU during the last two decades in order to reduce parenteral catheter-related sepsis, nutrition cost and translocation-induced multiple organ failure $^{(16,22)}$. Even if technical problems have limited its application, early EN is the recommended method of artificial feeding in ICU patients and some recent data suggest that EN is safe in patients with severe haemodynamic failure and that early EN could be an appropriate tool to reduce energy deficit and to improve ICU outcome $e^{(4,7,23-25)}$. However, the individual fluctuations in REE of ICU patients and the inaccuracy of predictive formulas used to evaluate REE complicate the assessment of the energy requirements and do not facilitate feeding and its early administration by ICU practitioners $^{(3,4,10,26)}$. Consequently, there is a deficit of "energy input - REE' resulting in negative energy balance in surgical ICU patients, particularly those treated with mechanical ventilation and nourished by the enteral route $e^{(4,16,24,27)}$. In non-surgical critically ill mechanically ventilated patients, hypoenergetic feeding has been also frequently associated

Abbreviations: EN, enteral nutrition; ICU, intensive care unit; REE, resting energy expenditure.

* Corresponding author: Dr Christophe Faisy, Hôpital Européen Georges Pompidou, fax + 33 156093202, email christophe.faisy@egp.aphp.fr 
with inappropriate prescription rate and/or with inadequate delivery of the $\operatorname{EN}^{(4,28,29)}$. The causes and consequences of energy deficit have been investigated in medical mechanically ventilated patients who received, at least in part, both EN and parenteral nutrition ${ }^{(29,30)}$

The estimation of daily energy deficit requires indirect calorimetry because the usual predictive equations for estimating REE are based on individual anthropometric characteristics $^{(29,30)}$ However, indirect calorimetry, the gold standard for REE assessment, is not always applicable in severe ICU patients, needs costly equipment and technical expertise, is time consuming and is not available everywhere ${ }^{(30,31)}$. In a previous prospective study, we found that indirect calorimetry was not applicable in $43 \%$ of severe ICU patients because they had conditions invalidating calorimetric measurements $^{(31)}$. We developed and validated a simple and practicable REE equation incorporating static (weight, height) and dynamic biometric parameters (temperature, minute ventilation) that measure the very variable and rapidly changing REE in severe medical ICU patients ${ }^{(31,32)}$. The present study identifies the determinants of the energy balance, estimated from our equation, and its relationship to ICU outcome in severely ill patients requiring prolonged acute mechanical ventilation and receiving exclusive EN.

\section{Subjects and methods}

\section{Patients and study design}

We conducted an observational, retrospective study in the twenty-bed medical ICU of a tertiary teaching hospital. The retrospective design protected from the Hawthorne effect (a change in behaviour when clinicians or nurses are being observed $)^{(33)}$. Because of the observational design of the study, no local Institutional Review Board authorization was needed according to French bioethics laws. Adult patients admitted in the ICU over a 1-year period requiring endotracheal intubation and mechanical ventilation $<6 \mathrm{~h}$ after ICU admission and intubated for at least $7 \mathrm{~d}$ were eligible for analysis. The least duration of $7 \mathrm{~d}$ for intubation allowed selection of patients requiring prolonged acute mechanical ventilation $(\geqq 96 \mathrm{~h})^{(34)}$ and allowed a realistic nutritional survey requiring ventilator measurements for REE calculation to be performed. Patients who were not receiving EN because of the following contraindications were not selected for analysis: complete intestinal obstruction or ileus, intractable vomiting or severe diarrhoea, intestinal ischaemia, gastrointestinal haemorrhage, high-output enterocutaneous fistulas ${ }^{(7,35)}$. We performed a systematic nutritional survey (estimation of REE, energy prescribed and delivered) from ICU admission to day 14 in order to focus our investigations on the level of early energy balance and its impact on outcome. Nutritional survey ceased when invasive mechanical ventilation was stopped before day 14 (death or extubation) at which stage EN intakes became difficult to estimate accurately and the calculation of REE (requiring ventilator measurements) was not possible.

\section{Definitions}

Total energy prescribed was calculated from glucose infusions and exclusive EN prescriptions based on local protocol described later. Energy delivered by nurses included energy actually administered per day by enteral feeds and glucose infusions. Propofol was not used for continuous sedation in our ICU. Recommended energy corresponded to target feeding based on nutritional protocol described later. The daily REE (kcal per d) was retrospectively calculated with the following predictive equation ${ }^{(31,32)}$ : $(8 \times$ body weight $(\mathrm{kg}))+(14 \times$ height $(\mathrm{cm}))+(32 \times$ minute ventilation $($ litres $/ \mathrm{min}))+\left(94 \times\right.$ body temperature $\left.\left({ }^{\circ} \mathrm{C}\right)\right)-4834$. No stress factor modifications were required ${ }^{(31)}$. Energy values were then given in $\mathrm{kJ}$ using the conversion factor $1 \mathrm{kcal}=4 \cdot 184 \mathrm{~kJ}$. Body weight, minute ventilation and body temperature were assessed from daily observational charts as described later. Height, assessed with measuring tape and the patient lying in a supine position, was collected from the medical file. Energy balance $(\mathrm{kJ}$ per $\mathrm{d}$ of mechanical ventilation) was calculated as follows: energy delivered calculated REE. The energy stored (adipose tissues, intramuscular TAG, and blood fatty acids or TAG) was ignored for the calculation of energy balance ${ }^{(2,4,24)}$. Energy deficit was assumed to correspond to a negative energy balance.

\section{Exclusive enteral nutrition}

EN was started within $24 \mathrm{~h}$ of ICU admission when gastrointestinal tract was functional ${ }^{(36)}$ and patients were haemodynamically stable. Patients received polymeric isoenergetic solution (Sondalis Iso ${ }^{\circledR}$; Nestlé Clinical Nutrition, France; $4.184 \mathrm{~kJ} / \mathrm{ml}, 50 \%$ carbohydrates, $35 \%$ lipids, $15 \%$ proteins). EN was delivered by continuous, pump-driven infusion via a nasogastric tube. Post-pyloric delivery was not performed. The target feeding recommended by the Société de Réanimation de Langue Française was $125.5 \mathrm{~kJ} / \mathrm{kg}$ (30 kcal $/ \mathrm{kg}$ ) per $\mathrm{d}$ (or per $\mathrm{kg}$ ideal body weight per $\mathrm{d}$ if $\mathrm{BMI}$ was $\left.>25 \mathrm{~kg} / \mathrm{m}^{2}\right)^{(36)}$. Parenteral nutrition was not used to compensate for insufficient amount of energy in our ICU. The gastric position of the tube was checked before the onset of feeding, and later by daily chest radiography. EN was to be started within $24 \mathrm{~h}$ of ICU admission at $20 \mathrm{ml} / \mathrm{h}$, and increased as previously reported $^{(37)}$, in the absence of gastrointestinal intolerance, until the recommended target energy was reached. Occurrence of gastrointestinal intolerance (diarrhoea, vomiting, abdominal distension or ileus) necessitated reducing the feeding rate by $50 \%^{(16,21,25)}$. EN was stopped in the event of procedures out of ICU or, if required, in ICU, and in case of persistence of severe gastrointestinal intolerance and then restarted after recovery. Gastric residual volumes were not routinely measured and gastric prokinetic drugs and stress ulcer prophylaxis were not systematically used in our $\operatorname{ICU}^{(21,38)}$. The gastric lumen tube was systematically washed with $20 \mathrm{ml}$ sterile water after administration of drugs by this route. In our unit, intubated patients were maintained with head-of-bed $>30^{\circ}$ to reduce the risk of aspiration ${ }^{(39)}$. EN was not discontinued when the prone position was used in patients with acute respiratory distress syndrome or when ventilator weaning test procedures were achieved.

\section{Patient data}

Baseline demographic data at the time of ICU admission included age, height, weight, main diagnosis and Simplified Acute Physiology Score II (SAPS II). The diagnosis of 
septic shock was retained in the presence of documented infection including bacteraemia, urinary tract infection and pneumonia $^{(39)}$. On each day of mechanical ventilation, the following data were retrospectively collected from charts prospectively completed by nurses: weight measured with a mobile electronic scale, temperature (mean of four values measured electronically in the ear at $6 \mathrm{~h}$ intervals during the day) and minute ventilation (mean of four values determined with the respiratory device at $6 \mathrm{~h}$ intervals during the day). We assessed from charts the total duration of EN interruptions (expressed as percentage of survey time) due to gastrointestinal intolerance (vomiting, severe diarrhoea, ileus) or diagnostic and therapeutic procedures. We also estimated the total length (percentage of survey time) of conditions that could decrease nutrient absorption or limit the volume of feeding rate prescription ${ }^{(16,19-21,40)}$ : use of sedatives, opioids, neuromuscular blocking agents or vasopressors, and presence of renal failure (defined as creatinine clearance $<50 \mathrm{ml} / \mathrm{min}$ ) with fluid overload.

\section{Statistical analysis}

Results were expressed as means with their standard errors for quantitative variables and numbers $(n)$ or percentages (\%) for categorical variables. Paired $t$ tests, Mann-Whitney or Fisher's tests were applied for comparison of relevant variables. Logistic regression was used for multivariate analysis. Relationships between ICU mortality and quartiles of mean energy balance were assessed with the Mantel-Haenszel test. A receiver operating characteristics curve was generated with a non-parametric method (JLABROC4 Eng Software ${ }^{\circledR}$; Johns Hopkins University, Baltimore, MD, USA) to determine the best operating point as mean energy deficit threshold for predicting ICU mortality. ICU survival was analysed by Kaplan-Meier curves, and comparison between groups was performed by the log-rank test. Statistics were calculated with the StatView ${ }^{\circledR} 4.5$ (Abacus Concept Inc., Berkeley, CA, USA) and EPI INFO 3.4 (Centers for Disease Control and Prevention, Atlanta, GA, USA) software. We considered a difference to be significant when the $\alpha$ risk was $<5 \%(P<0.05)$.

\section{Results}

Patients

Forty-two patients out of 684 consecutive patients $(6 \%)$ admitted to our ICU were eligible for analysis over a 1 -year period. Among non-eligible patients, 198 were not intubated during their ICU stay, 444 were intubated $>6 \mathrm{~h}$ after ICU admission or intubated $<7 \mathrm{~d}$ or had contraindication for EN during acute mechanical ventilation. Four eligible patients were excluded because of incomplete data or missing files. Finally, thirty-eight patients were included in the nutrition survey. Main diagnoses at ICU admission were: septic shock ( $n$ 19), acute respiratory failure due to congestive heart failure (n 11), acute respiratory distress syndrome $(n 4)$ and nontraumatic coma ( $n$ 4); characteristics and demographic data are summarized in Table 1.
Table 1. Description of the patients (n 38) requiring prolonged acute mechanical ventilation

(Mean values with their standard errors)

\begin{tabular}{lcc}
\hline & Mean & SEM \\
\hline Age (years) & $67 \cdot 1$ & $2 \cdot 4$ \\
Males $(n)$ & 21 & \\
Females $(n)$ & 17 & \\
Length of hospital stay before ICU admission (d) & $6 \cdot 1$ & $1 \cdot 7$ \\
SAPS II at ICU admission & $46 \cdot 5$ & $2 \cdot 0$ \\
Height at ICU admission (cm) & $167 \cdot 1$ & $1 \cdot 0$ \\
Weight at ICU admission (kg) & $71 \cdot 6$ & $3 \cdot 2$ \\
Length of ICU stay (d) & $28 \cdot 0$ & $3 \cdot 4$ \\
Length of invasive ventilation (d) & $23 \cdot 4$ & $2 \cdot 9$ \\
ICU death & & \\
$n$ & 27 & \\
$\%$ & 72 & \\
\hline
\end{tabular}

ICU, intensive care unit; SAPS II, Simplified Acute Physiology Score II.

\section{Energy balance}

Mean prescribed, delivered and recommended energies, mean calculated REE and mean energy balance are summarized in Table 2. Mean energy deficit was stable from day 3 to day 14 of mechanical ventilation (Fig. 1 (A)). The ratio of delivered/prescribed energy was between 60 and $70 \%$ during the first $14 \mathrm{~d}$ of mechanical ventilation whereas prescribed energy/estimated REE ratio remained close to $40 \%$ from day 3 (Fig. 1 (B)). The total duration of $\mathrm{EN}$

Table 2. Mean energy/d of mechanical ventilation, and presence of conditions limiting feeding administration or feeding prescription in the first $14 \mathrm{~d}$ of respiratory support in patients $(n 38)$ requiring prolonged acute mechanical ventilation $\ddagger$

(Mean values with their standard errors)

\begin{tabular}{lcr}
\hline Energy (per d of mechanical ventilation) & Mean & SEM \\
\hline Prescribed energy & & \\
kJ & $4176^{\star \star \star} \dagger \dagger \dagger$ & 209 \\
kcal & $998^{\star \star \star} \dagger \dagger \dagger$ & 50 \\
Delivered energy & & \\
kJ & $2946^{\star \star \star}$ & 176 \\
kcal & $704^{\star \star \star}$ & 42 \\
Recommended energy (36) & & \\
kJ & $9234^{\star \star} \dagger \dagger \dagger$ & 393 \\
kcal & $2207^{\star \star} \dagger \dagger \dagger$ & 94 \\
Calculated REE ${ }^{(31)}$ & & \\
kJ & 8364 & 142 \\
kcal & 1999 & 34 \\
Energy balance§ & & 222 \\
kJ & -5439 & 53 \\
kcal & 1300 & $3 \cdot 4$ \\
Percentage of time with & & $5 \cdot 8$ \\
EN interruptions & $22 \cdot 9$ & $5 \cdot 3$ \\
Sedatives & $64 \cdot 0$ & $4 \cdot 2$ \\
Opioids & $62 \cdot 2$ & $6 \cdot 8$ \\
Neuromuscular blocking agents & $9 \cdot 8$ & $7 \cdot 9$ \\
Vasopressors & $54 \cdot 3$ & $50 \cdot 7$ \\
Renal failure with fluid overload & & \\
\hline
\end{tabular}

EN, enteral nutrition; REE, resting energy expenditure.

Mean values were significantly different from those of the calculated REE: ${ }^{\star \star} P<0.01,{ }^{\star \star \star} P<0.0001$

Mean values were significantly different from those of the delivered energy (paired $t$ test): $\dagger \dagger \dagger P<0.0001$.

$\ddagger$ For details of subjects and procedures, see the Subjects and methods section and Table 1.

$\S$ Energy balance $=$ delivered energy - calculated REE 
(A)

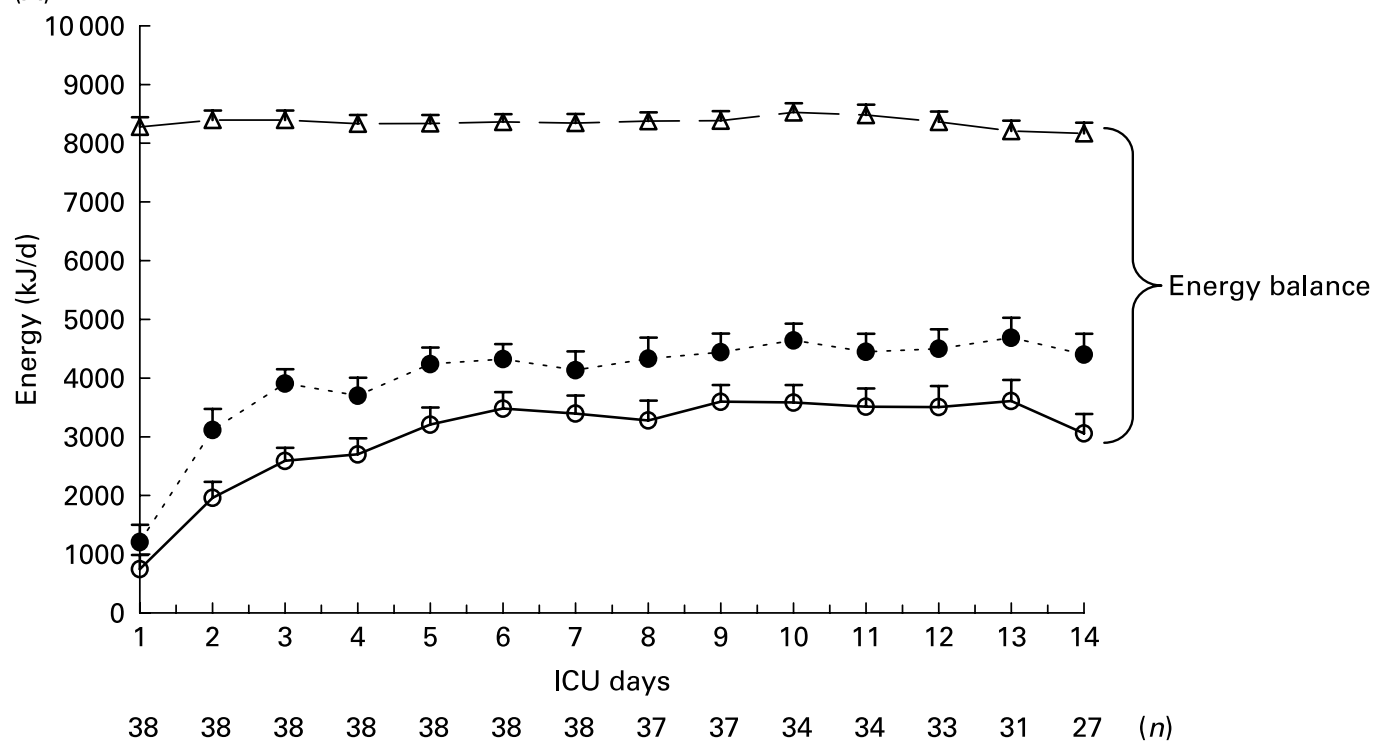

(B)

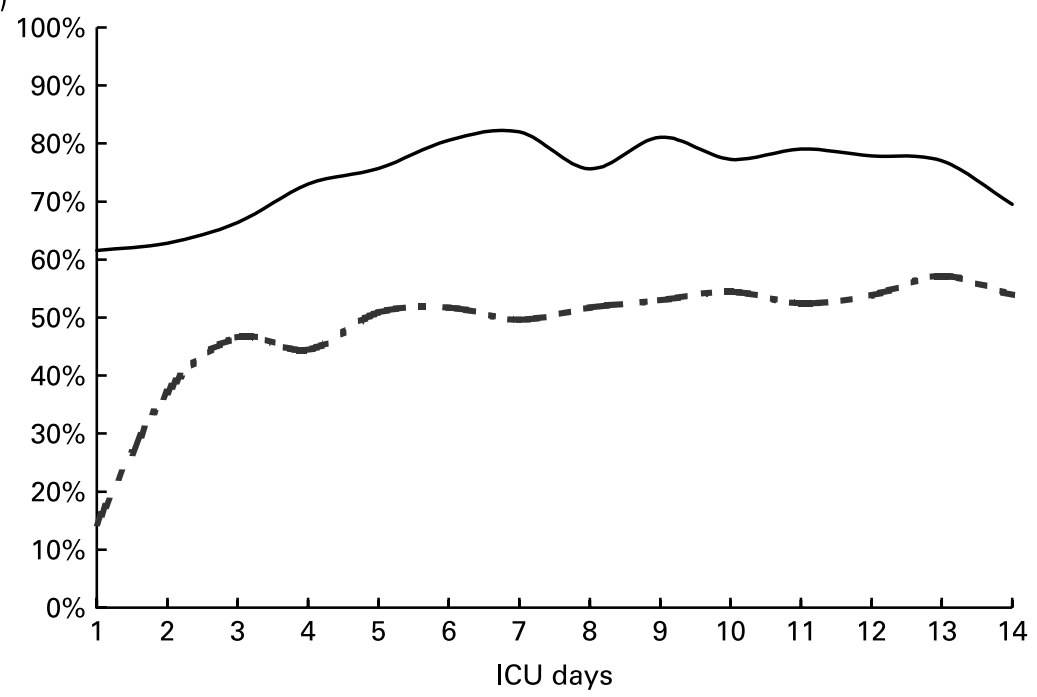

Fig. 1. (A), Evolution of prescribed energy $(\bullet)$, delivered energy $(O)$ and resting energy expenditure (REE) calculated with the Faisy 2003 equation ${ }^{(31)}(\triangle)$ in patients requiring prolonged acute mechanical ventilation $(n 38)$. Energy balance $=$ delivered energy - calculated REE. Values are means with their standard errors depicted by vertical bars. (B), Delivered/prescribed (-) and delivered/calculated REE energy ratios (--).

interruptions and the length of conditions limiting feeding absorption or feeding rate prescription for the first $14 \mathrm{~d}$ of mechanical ventilation are summarized in Table 2 . The main causes of EN interruptions were diagnostic procedures in or out of ICU and vomiting.

\section{Impact of energy deficit on intensive care unit outcome}

Non-survivors had higher mean energy deficit than ICU survivors (Table 3). Furthermore, non-survivors had a daily energy deficit higher than ICU survivors and that difference was statistically significant from day 10 of mechanical ventilation (Fig. 2). Non-survivors were similar to survivors for demographic characteristics, causes of ICU admission, factors influencing feeding administration or feeding prescription but spent more time sedated (Table 3). Logistic regression analysis, performed with factors influencing ICU outcome, identified mean energy deficit as independently associated with ICU death (Table 4). By the Mantel-Haenszel test, there was an increasing trend in ICU mortality across the quartiles of mean energy deficit estimated in the first $14 \mathrm{~d}$ of mechanical ventilation (Fig. 3 (A)). We used the best operating point of the receiver operating characteristics curve (Fig. 3 (B)) of mean energy deficit to determine a threshold of $5021 \mathrm{~kJ}(1200 \mathrm{kcal})$ per d of mechanical ventilation for predicting ICU death after the fourteenth ICU day (sensitivity $80 \%$, specificity $65 \%$, OR 6.12 (95\% CI $1.33,28.2$ ), positive likelihood ratio 2.28). Twenty-five patients had a mean energy deficit $\geqq 5021 \mathrm{~kJ}(1200 \mathrm{kcal})$ per d of mechanical ventilation and they had a higher ICU mortality rate than patients with lower mean energy deficit $(n$ 13) after the fourteenth ICU day (Fig. 4). 
Table 3. Characteristics and conditions limiting feeding administration or feeding prescription in the first $14 \mathrm{~d}$ of respiratory support in intensive care unit (ICU) deaths and in ICU survivors who experienced prolonged acute mechanical ventilationł

(Mean values with their standard errors)

\begin{tabular}{|c|c|c|c|c|c|}
\hline & \multicolumn{2}{|c|}{ ICU deaths ( $n$ 27) } & \multicolumn{2}{|c|}{ ICU survivors $(n 11)$} & \multirow[b]{2}{*}{$P \S$} \\
\hline & Mean & SEM & Mean & SEM & \\
\hline Age (years) & $66 \cdot 0$ & $3 \cdot 0$ & 69.8 & $3 \cdot 6$ & 0.44 \\
\hline BMl $\left(\mathrm{kg} / \mathrm{m}^{2}\right)$ & $25 \cdot 5$ & 1.4 & $25 \cdot 9$ & $2 \cdot 2$ & 0.75 \\
\hline Male/female $(n)$ & $14 / 13$ & & $7 / 4$ & & 0.72 \\
\hline \multicolumn{6}{|l|}{ Cause of ICU admission $(n)$} \\
\hline Septic shock & 14 & & 5 & & 0.40 \\
\hline Congestive heart failure & 6 & & 5 & & \\
\hline Acute respiratory distress syndrome & 3 & & 1 & & \\
\hline Non-traumatic coma & 4 & & 0 & & \\
\hline Length of hospital stay before ICU (d) & $7 \cdot 3$ & $2 \cdot 3$ & $3 \cdot 1$ & $1 \cdot 0$ & 0.72 \\
\hline Length of ICU stay (d) & $27 \cdot 5$ & 4.5 & $29 \cdot 1$ & 3.5 & 0.17 \\
\hline Length of invasive ventilation (d) & $25 \cdot 4$ & 3.9 & $18 \cdot 3$ & $2 \cdot 6$ & 0.24 \\
\hline \multicolumn{6}{|c|}{ Mean energy deficit (per $d$ of mechanical ventilation) } \\
\hline kJ & 5832 & 230 & 4481 & 410 & 0.0004 \\
\hline kcal & 1394 & 55 & 1071 & 98 & 0.0004 \\
\hline \multicolumn{6}{|l|}{ Percentage of time with } \\
\hline EN interruptions & $20 \cdot 9$ & $3 \cdot 7$ & $27 \cdot 7$ & $7 \cdot 4$ & 0.38 \\
\hline Sedatives & 71.4 & $7 \cdot 1$ & 45.9 & 7.5 & 0.04 \\
\hline Opioids & $67 \cdot 8$ & $6 \cdot 2$ & 48.5 & $9 \cdot 6$ & 0.13 \\
\hline Neuromuscular blocking agents & $13 \cdot 8$ & $5 \cdot 7$ & 0.0 & 0.0 & 0.21 \\
\hline Vasopressors & $61 \cdot 3$ & $7 \cdot 7$ & $37 \cdot 0$ & $12 \cdot 6$ & 0.15 \\
\hline Renal failure with fluid overload & $57 \cdot 9$ & $9 \cdot 4$ & $33 \cdot 0$ & $14 \cdot 1$ & 0.16 \\
\hline
\end{tabular}

EN, enteral nutrition; SAPS II, Simplified Acute Physiology Score II.

¥For details of subjects and procedures, see the Subjects and methods section and Table 1.

$\S$ Statistical analysis was by Mann-Whitney test for quantitative variables and by Fisher's test for categorical variables (Male/female and Cause of ICU admission).

\section{Discussion}

The present study shows that negative energy balance, calculated with a predictive estimation of REE based on static and dynamic biometric parameters, is associated with ICU mortality in the most severely ill medical patients requiring acute prolonged mechanical ventilation and receiving exclusive EN. Our predictive method was applicable to the ICU patients measured in the current study and the present results are in agreement with recent works performed with calorimetric devices confirming the usefulness of our predictive equation when indirect calorimetry is not available or inapplicable ${ }^{(29,32)}$. However, the magnitude of the deficit between estimated energy requirements and measured energy intakes was unexpected considering the procedures used to enable adequate feeding. The present result may be explained, at least in part, by the inclusion of older and very seriously ill patients with a high incidence of clinical situations pre-disposing to gastroparesis or interruption of feeding.

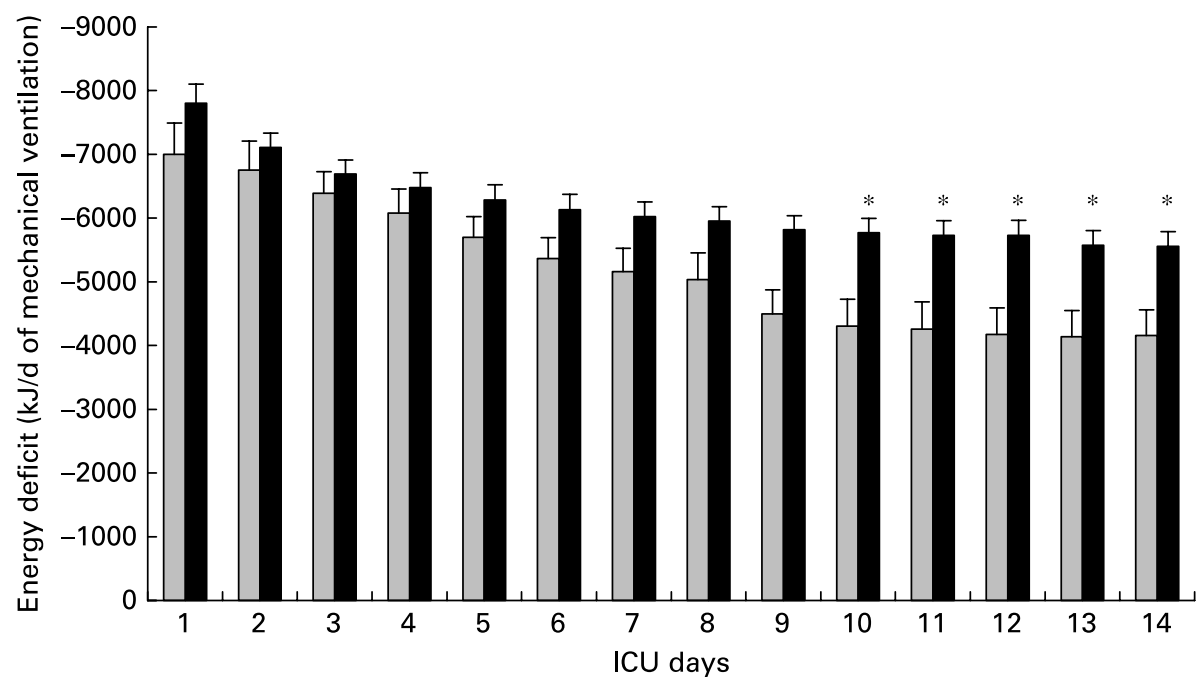

Fig. 2. Evolution of daily energy deficit of intensive care unit (ICU) survivors ( $\square ; n 11)$ and of ICU deaths ( $\square$; $n 27)$ in patients requiring prolonged acute mechanical ventilation. Values are means with their standard errors depicted by vertical bars. Mean values were significantly different from those of the ICU survivors: ${ }^{*} P<0.05$. 
Table 4. Logistic model coefficients table for intensive care unit (ICU) death in patients requiring prolonged acute mechanical ventilation (multivariate analysis) $\ddagger$

\begin{tabular}{|c|c|c|c|c|}
\hline & Coefficient & SEM & $x^{2}$ & $P$ \\
\hline Length of invasive ventilation (d) & 0.04 & 0.05 & 0.63 & 0.42 \\
\hline SAPS II at ICU admission & 0.08 & 0.05 & 2.55 & $0 \cdot 11$ \\
\hline $\begin{array}{l}\text { Mean energy deficit } \\
(\mathrm{kJ} / \mathrm{d} \text { of mechanical ventilation)§ }\end{array}$ & $7 \cdot 92$ & 3.50 & $5 \cdot 11$ & 0.02 \\
\hline Time sedated (\% first $14 \mathrm{~d})$ & 2.52 & 1.53 & 2.69 & 0.10 \\
\hline $\begin{array}{l}\text { Time with opioids } \\
\text { (\% first } 14 \mathrm{~d})\end{array}$ & -3.85 & $3 \cdot 81$ & 1.02 & 0.31 \\
\hline $\begin{array}{l}\text { Time with vasopressors } \\
\text { (\% first } 14 \mathrm{~d})\end{array}$ & $2 \cdot 09$ & $2 \cdot 74$ & 0.58 & 0.44 \\
\hline $\begin{array}{l}\text { Time with renal failure } \\
\text { (\% first } 14 \mathrm{~d})\end{array}$ & -0.03 & $1 \cdot 23$ & 0.001 & 0.98 \\
\hline
\end{tabular}

SAPS II, Simplified Acute Physiology Score II.

$\ddagger$ For details of subjects and procedures, see the Subjects and methods section and Table 1.

$\S$ Mean energy deficit was independently associated with ICU death in patients requiring prolonged acute mechanical ventilation.

Moreover, the retrospective observational design of the present study, by limiting the Hawthorne effect, underlines the gap between evidence and practice ('the true life') for EN support in critically ill patients ${ }^{(21,33,41)}$.

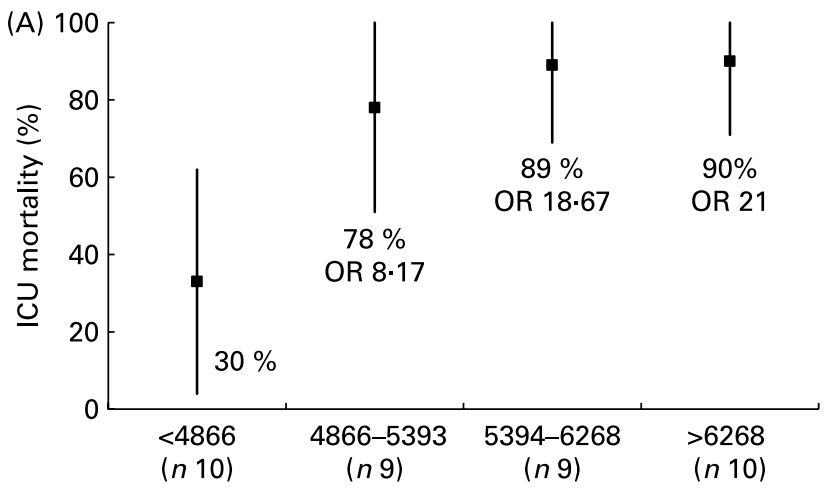

Energy deficit ( $\mathrm{kJ} / \mathrm{d}$ of mechanical ventilation)

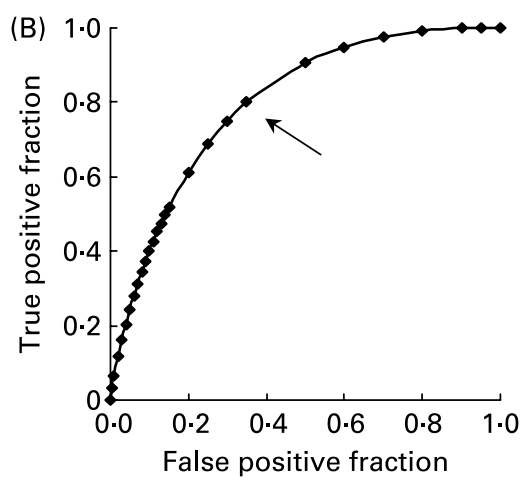

Fig. 3. (A), Relationship between intensive care unit (ICU) mortality and quartiles of mean energy deficit in the first $14 \mathrm{~d}$ in patients requiring prolonged acute mechanical ventilation. Values are percentages with $95 \% \mathrm{Cl}$ depicted by vertical bars. OR, comparison $v$. the first quartile. Values were significantly different from that of the first quartile (Mantel-Haenszel test): $P=0.003$. (B), Receiver operating characteristics (ROC) curve of mean energy deficit for ICU mortality. The area under the ROC curve is 0.80 (SEM 0.08). $\leftarrow, 5021 \mathrm{~kJ}(1200 \mathrm{kcal})$ per d.

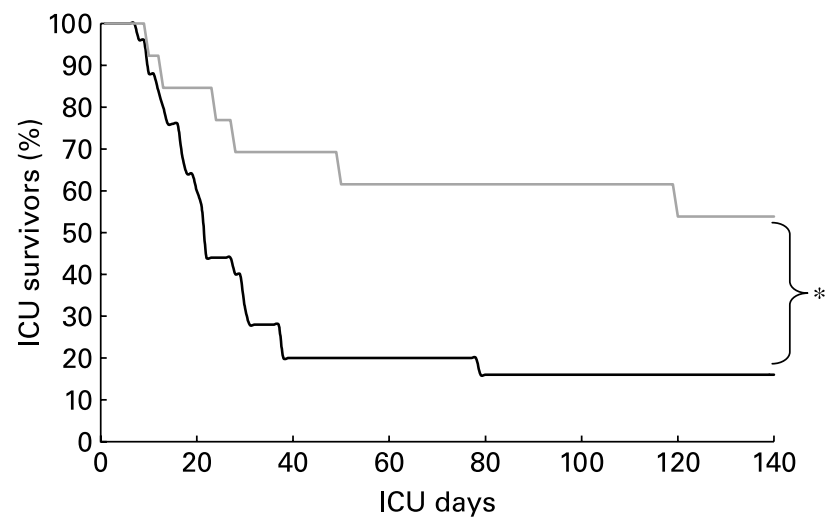

Fig. 4. Kaplan-Meier analysis of intensive care unit (ICU) survival rate in patients with mean energy deficit $\geqq 5021 \mathrm{~kJ}(1200 \mathrm{kcal}) / \mathrm{d}$ of mechanical ventilation (-; $n$ 25) and with mean energy deficit $<5021 \mathrm{~kJ}(1200 \mathrm{kcal}) / \mathrm{d}$ of mechanical ventilation (-; $n$ 13). ${ }^{*}$ Values were significantly different $(P=0.01$; log-rank test).

Also, unexpectedly, we found that estimated REE remained stable during the first $14 \mathrm{~d}$ of mechanical ventilation instead of a hypometabolic 'ebb' phase during the first 24-48h, followed by 'flow' phase ${ }^{(42)}$. Other investigators described similar classic evolution in critically ill subjects by using indirect calorimetry to estimate REE but the limitations of this method in unstable patients were not considered ${ }^{(19,31)}$. Nevertheless, methodological biases in previous studies do not appear sufficient to explain the absence of significant change in REE in the present study. It is likely that many patients were already in the 'flow' phase by the time they were admitted to our ICU and the modern fluid resuscitation would shorten the period of hypotension resulting in a short 'ebb' phase. In addition, our patients were frequently infected and/or shocked at ICU admission and metabolic alterations in sepsis (elevated REE) associated with metabolic overstimulation caused by inotropic-vasopressor agents could also explain the absence of early hypometabolism, increasing energy deficit in the present study ${ }^{(7,15,31)}$.

Recent studies of energy deficit in severely ill patients estimated the prescribed/REE energy ratio close to $70 \%$ with relatively great variability ${ }^{(2,5,7,21,24,29,43)}$ compared to $40 \%$ after the third day in the current study. The difference between the intakes recommended by French guidelines and our REE estimation using the Faisy 2003 equation possibly suggests that the former are not adapted for the patients studied. Moreover, the energy deficit compared to the Faisy equation is large, suggesting that protocols for ensuring adequate EN are not successful. The causes of delayed or underdelivered EN are well known: gastro-intestinal intolerance enhanced by a high rate of splanchnic $\mathrm{O}_{2}$ extraction in patients with shock or cardiac failure ${ }^{(16,24)}$; drugs such as sedatives and vasopressors resulting in gastroparesis ${ }^{(7,16,40)}$; various diagnostic or therapeutic procedures, especially in mechanically ventilated patients ${ }^{(17,24,29,43)}$; lack of medical and paramedical management of feeding, particularly the delays in restarting EN after procedures ${ }^{(5,7,18,28,43,44)}$. Our acute mechanically ventilated patients frequently had compromised haemodynamic status at ICU admission and most of them received prolonged infusion of vasopressors and sedatives. 
These conditions were also present in other studies but without the large energy deficit found in the current study ${ }^{(4,21,24)}$. One explanation may be the high incidence of renal failure in our patients necessitating limitation of feed volume to limit fluid overload. Moreover, similar to previous studies, $71 \%$ of the energy prescribed was delivered suggesting that energy deficit was not mostly caused by nursing practices $(5,7,16,27,29,44)$. Additionally, the duration of EN interruptions for procedures or severe gastrointestinal intolerance was close to $23 \%$ of the total time of survey and explained most of the differences between delivered and prescribed energy. We did not routinely use post-pyloric feeding, prokinetic drugs administration or measurement of gastric residual volumes to manage poor gastric emptying as their use is controversial and can involve premature cessation or delayed $\mathrm{EN}^{(5,7,41,45-47)}$. We merely raised the patient's head and shoulders ${ }^{(44,48)}$, questioning this practice. We conclude that underestimation of energy requirements, delay in starting or increasing EN, and overprecautionary interruptions in EN resulted in underfeeding in our ICU patients ${ }^{(7,43,44)}$. In addition, herein, we found that the percentage of time with situations limiting feed administration was higher than periods with EN interruptions, suggesting the inappropriate medical practices were the major cause of the large negative energy balance reported in the present study. Contrary to recent prospective studies, our survey suggests that implementation of a standardized nutritional management protocol in ICU does not prevent such conservative prescribing patterns ${ }^{(7,41)}$. The lack of consensus concerning the energy requirement for critically ill patients and the uncertainty about the safety of early EN in mechanically ventilated patients may explain this conservative approach ${ }^{(5,7,26,27,43)}$. However, the present study indicates that the gap between recommended nutrition care and practice regarding enteral feeding still exists and it results from lack of knowledge and interest of the importance of nutritional assessment by nurses and doctors ${ }^{(49-51)}$. Confusion between medical and nursing staff over responsibility for $\mathrm{EN}$ probably plays a part in underestimation of energy requirements in $\mathrm{ICU}^{(52)}$. Importantly, no qualified dietitian was regularly present in our ICU while recent surveys emphasized that doctors and nurses supported by clinical dietitians had better focus on clinical nutrition, especially in daily ICU practice ${ }^{(53,54)}$. Taken together, the present findings highlight the causes of the discrepancy between recommended and prescribed or delivered energy in our severe ICU patients. Moreover, they also underline the limitations of written standardized nutritional protocols and the relevance of a nutritional support team for improving energy balance of the most severely injured patients receiving EN.

We showed that negative energy balance during the first $14 \mathrm{~d}$ in the ICU is an independent factor of ICU mortality in patients requiring acute prolonged mechanical ventilation. Moreover, the present results confirm recent findings indicating that length of acute mechanical ventilation does not influence ICU discharge ${ }^{(34)}$. It has been shown that early energy deficit is strongly correlated with infectious complications and organ failures in surgical and medical ICU patients $^{(4,24,30)}$. However, correlations do not mean causality and the relationships between ICU mortality and energy deficit were not clearly evidenced in these studies. Interestingly, in the present study the patients who died, unlike survivors, continued to have a large negative energy balance $\geq 5021 \mathrm{~kJ}$ ( $1200 \mathrm{kcal})$ per $\mathrm{d}$ after the first week of mechanical ventilation. Taken together, the present findings suggest that energy deficit contributes to ICU mortality and that this deficit results from persistent hypermetabolism combined with inadequate nutrient intake for $>1$ week of mechanical ventilation. This highlights the possibility that ICU mortality could be reduced by ensuring the energy deficit is less than $5021 \mathrm{~kJ}(1200 \mathrm{kcal})$ per day for the first 2 weeks of mechanical ventilation. Alternative routes of feeding (post-pyloric or parenteral) should be considered if EN via a nasogastric tube failed to achieve this goal. Furthermore, assessment of body composition could be a useful means to detect and prevent the effects of underfeeding in ICU patients ${ }^{(55)}$.

Further investigation to confirm the present findings is required before extrapolating the present results beyond our patient population and procedures of enteral feeding. However, it is important to keep in mind that energy deficit may impact on outcome mainly in the most severely ill ICU patients and progress in nutritional assessment of this 'targeted' population could improve significantly their ICU survival. The present results support this hypothesis confirming the significant impact of negative energy balance on outcome and highlighting the causes of underfeeding in the very sick medical ICU population. We expect the present results could contribute to develop better focus on nutrition assessment practices in the most severely injured patients for whom the impact of negative energy balance is clinically relevant. Our nutrition practices were modified on the basis of the present study, especially special attention for the most severely ill patients with prolonged acute mechanical ventilation.

In summary, the present study shows that large, negative energy balance occurs during acute prolonged mechanical ventilation even in the presence of standardized nutritional management protocol. Energy deficit seems to be an independent determinant of ICU mortality and its causes, under prescription and interruption of feeding, must be addressed through an educational policy. Specifically, feed prescription based on accurate REE estimation appears to be a prerequisite for improving nutritional management of ICU patients. In this way, the use of our simple predictive estimation of REE based on static and dynamic biometric parameters could be an alternative approach when indirect calorimetry is not available or inapplicable.

\section{Acknowledgements}

The authors appreciate the diligent revision of this manuscript by Dr Stephen J. Taylor, from the Department of Nutrition and Dietetics, Frenchay Hospital, Bristol, UK. No author has any financial or personal interest in any company or organization relevant to the field of research in connection with this work. The funding source was Service de Réanimation Médicale, Hôpital Européen Georges Pompidou, Paris, France. This study was not sponsored by gifts or fellowships. C. F. and F. D. were responsible for the conception and design of the study, data collection and analysis, and drafting the report. N. L. and J.-F. S. were responsible for interpretation of the data and drafting the report. I. A., J.-M. T. and J.-Y. F. were responsible for revising the report. 


\section{References}

1. Wray CJ, Mammen JM \& Hasselgren PO (2002) Catabolic response to stress and potential benefits of nutrition support. Nutrition 18, 971-977.

2. Plank LD \& Hill GL (2003) Energy balance in critical illness. Proc Nutr Soc 62, 545-552.

3. Reid CL (2004) Nutritional requirements of surgical and critically-ill patients: do we really know what they need? Proc Nutr Soc 63, 467-472.

4. Villet S, Chiolero RL, Bollmann MD, Revelly JP, Cayeux RNM, Delarue J \& Berger M (2005) Negative impact of hypocaloric feeding and energy balance on clinical outcome in ICU patients. Clin Nutr 24, 502-509.

5. Reid C (2006) Frequency of under- and overfeeding in mechanically ventilated ICU patients: causes and possible consequences. J Hum Nutr Diet 19, 13-22.

6. Giner M, Laviano A, Meguid MM \& Gleason JR (1996) In 1995 a correlation between malnutrition and poor outcome in critically ill patients still exists. Nutrition 12, 23-29.

7. Barr J, Hecht M, Flavin KE, Khorana A \& Gould MK (2004) Outcomes in critically ill patients before and after the implementation of an evidence-based nutritional management protocol. Chest 125, 1446-1457.

8. Rubinson L, Diette GB, Song X, Brower RG \& Krishnan JA (2004) Low caloric intake is associated with nosocomial bloodstream infections in patients in the medical intensive care unit. Crit Care Med 32, 350-357.

9. Pingleton SK (2001) Nutrition in chronic critical illness. Clin Chest Med 22, 149-163.

10. McClave SA, Lowen CC, Kleber MJ, Nicholson JF, Jimmerson SC, McConnell JW \& Jung L (1998) Are patients fed appropriately according to their caloric requirements? J Parenter Enteral Nutr 22, 375-381.

11. Huang $\mathrm{YC}$, Yen $\mathrm{CE}$, Cheng $\mathrm{CH}$, Jih $\mathrm{KS} \&$ Kan $\mathrm{MN}$ (2000) Nutritional status of mechanically ventilated critically ill patients: comparison of different types of nutritional support. Clin Nutr 19, 101-107.

12. Galban C, Montejo JC, Mesejo A, Marco P, Celaya S, SanchezSegura JM, Farre M \& Bryg DJ (2000) An immune-enhancing enteral diet reduces mortality rate and episodes of bacteremia in septic intensive care unit patients. Crit Care Med 28, 643-648.

13. Rodriguez L (2004) Nutritional status: assessing and understanding its value in the critical care setting. Crit Care Nurs Clin North Am 16, 509-514.

14. Chiolero R, Revelly JP \& Tappy L (1997) Energy metabolism in sepsis and injury. Nutrition 13, 45S-51S.

15. Trager K, DeBacker D \& Radermacher P (2003) Metabolic alterations in sepsis and vasoactive drug-related metabolic effects. Curr Opin Crit Care 9, 271-278.

16. Berger MM, Chiolero RL, Pannatier A, Cayeux MC \& Tappy L (1997) A 10-year survey of nutritional support in a surgical ICU: 1986-1995. Nutrition 13, 870-877.

17. Rice TW, Swope T, Bozema S \& Wheeler AP (2005) Variation in enteral nutrition delivery in mechanically ventilated patients. Nutrition 21, 786-792.

18. O'Leary-Kelley CM, Puntillo KA, Barr J, Stotts N \& Douglas MK (2005) Nutritional adequacy in patients receiving mechanical ventilation who are fed enterally. Am J Crit Care 14, 222-231.

19. Uehara M, Plank LD \& Hill GL (1997) Components of energy expenditure in patients with severe sepsis and major trauma: a basis for clinical care. Crit Care Med 27, 1295-1302.

20. Mentec H, Dupont H, Bocchetti M, Cani P, Ponche F \& Bleichner G (2001) Upper digestive intolerance during enteral nutrition in critically ill patients: frequency, risk factors, and complications. Crit Care Med 29, 1955-1961.
21. De Jonghe B, Appere-De-Vechi C, Fournier M, Tran B, Merrer J, Melchior JC \& Houtin H (2001) A prospective survey of nutritional support practices in intensive care unit patients: what is prescribed? What is delivered? Crit Care Med 29, 8-12.

22. Heyland DK, MacDonald S, Keefe L \& Drover JW (1998) Total parenteral nutrition in the critically ill patient: a meta-analysis. JAMA 280, 2013-2019.

23. Jolliet P, Pichard C, Biolo G, et al. (1999) Enteral nutrition in intensive care patients: a practical approach. Clin Nutr 18, 47-56.

24. Berger MM, Revelly JP, Cayeux MC \& Chiolero RL (2005) Enteral nutrition in critically ill patients with severe hemodynamic failure after cardiopulmonary bypass. Clin Nutr $\mathbf{2 4}$, 124-132.

25. Taylor SJ, Fettes SB, Jewkes C \& Nelson RJ (1999) Prospective, randomized, controlled trial to determine the effect of early enhanced enteral nutrition on clinical outcome in mechanically ventilated patients suffering head injury. Crit Care Med 27, 2525-2531.

26. Ibrahim EH, Mehringer L, Prentice D, Sherman G, Schaiff R, Fraser V \& Kollef MH (2002) Early versus late enteral feeding of mechanically ventilated patients: results of a clinical trial. J Parenter Enteral Nutr 26, 174-181.

27. Dock-Nascimento DB, Tavares VM \& de Aguilar-Nascimento JE (2005) Evolution of nutritional therapy prescription in critically ill patients. Nutr Hosp 20, 343-347.

28. Adam S \& Batson S (1997) A study of problems associated with the delivery of enteral feed in critically ill patients in five ICUs in the UK. Intensive Care Med 23, 261-266.

29. Petros S \& Engelmann L (2006) Enteral nutrition delivery and energy expenditure in medical intensive care patients. Clin Nutr 25, 51-59.

30. Dvir D, Cohen J \& Singer P (2006) Computerized energy balance and complications in critically ill patients: an observational study. Clin Nutr 25, 37-44.

31. Faisy C, Guerot E, Diehl JL, Labrousse J \& Fagon JY (2003) Assessment of resting energy expenditure in mechanically ventilated patients. Am J Clin Nutr 78, 241-249.

32. Savard J, Faisy C, Lerolle N, Guérot E, Diehl JL \& Fagon JY (2008) Validation of a predictive method for an accurate assesment of resting energy expenditure in medical mechanically ventilated patients. Crit Care Med 36, 1175-1183.

33. Campbell JP, Maxey VA \& Watson WA (1995) Hawthorne effect: implications for prehospital research. Ann Emerg Med 26, 590-594.

34. Zilberberg MD, Luippold RS, Sulsky S \& Shorr AF (2008) Prolonged acute mechanical ventilation, hospital resource utilization, and mortality in the United States. Crit Care Med 36, 724-730.

35. American Society for Parenteral and Enteral Nutrition (1993) Guidelines for the use of parenteral and enteral nutrition in adult and pediatric patients. J Parenter Enteral Nutr 17, 1SA-52SA.

36. Thuong M \& Leteurtre S (2003) [Experts recommendations of the Société de Réanimation de Langue Française Enteral nutrition in critical care]. Réanimation 12, 350-354.

37. Genton L, Dupertuis YM, Romand JA, Simonet ML, Jolliet P, Huber O, Kudsk KA \& Picharg C (2004) Higher calorie prescription improves nutrient delivery during the first 5 days of enteral nutrition. Clin Nutr 23, 307-315.

38. Faisy C, Guerot E, Diehl JL, Iftimovici E \& Fagon JY (2003) Clinically significant gastrointestinal bleeding in critically ill patients with and without stress-ulcer prophylaxis. Intensive Care Med 29, 1306-1313.

39. Chastre J \& Fagon JY (2002) Ventilator-associated pneumonia. Am J Respir Crit Care Med 165, 867-903. 
40. Ravasco P \& Camilo ME (2003) Tube feeding in mechanically ventilated critically ill patients: a prospective clinical audit. Nutr Clin Pract 18, 427-433.

41. Heyland DK, Dhaliwal R, Day A, Jain M \& Drover J (2004) Validation of the Canadian clinical practice guidelines for nutrition support in mechanically ventilated, critically ill adult patients: results of a prospective observational study. Crit Care Med 32, 2260-2266.

42. Cuthbertson D (1942) Post-shock metabolic response. Lancet i, 433-437.

43. Kyle UG, Genton L, Heidegger CP, Maisonneuve N, Karsegard VL, Huber O, Mensi N, Romand JA, Jolliet P \& Pichard C (2006) Hospitalized mechanically ventilated patients are at higher risk of enteral underfeeding than non-ventilated patients. Clin Nutr 25, 727-735.

44. Marshall AP \& West SH (2006) Enteral feeding in the critically ill: are nursing practices contributing to hypocaloric feeding? Intensive Crit Care Nurs 22, 95-105.

45. Davies AR, Froomes PR, French CJ, Bellomo R, Gutteridge GA, Nyulasi I, Walker R \& Sewel RB (2002) Randomized comparison of nasojejunal and nasogastric feeding in critically ill patients. Crit Care Med 30, 586-590.

46. Reignier J, Bensaid S, Perrin-Gachadoat D, Burdin M, Boiteau R \& Tenaillon A (2002) Erythromycin and early enteral nutrition in mechanically ventilated patients. Crit Care Med 30, 1237-1241.

47. Montejo JC, Grau T, Acosta J, et al. (2002) Multicenter, prospective, randomized, single-blind study comparing the efficacy and gastrointestinal complications of early jejunal feeding with early gastric feeding in critically ill patients. Crit Care Med 30, 796-800.

48. Adam S (1994) Aspects of current research in enteral nutrition in the critically ill. Care Crit Ill 10, 246-251.

49. Lennard-Jones JE, Arrowsmith H, Davison C, Denham AF \& Micklewright A (1995) Screening by nurses and junior doctors to detect malnutrition when patients are first assessed in hospital. Clin Nutr 14, 336-340.

50. Wentzel Persenius M, Larsson BW \& Hall-Lord ML (2006) Enteral nutrition in intensive care. Nurses' perceptions and bedside observations. Intensive Crit Care Nurs 26, 82-94.

51. Lindorff-Larsen K, Hojgaard Rasmussen H, Kondrup J, Staun M \& Ladefoged K (2007) The Scandinavian Nutrition Group Management and perception of hospital undernutrition - a positive change among Danish doctors and nurses. Clin Nutr 26, 371-378.

52. Adam S \& Webb AR (1990) Attitudes to the delivery of enteral nutritional support to patients in British intensive care units. Clin Intensive Care 1, 150-156.

53. Fulbrook P, Bongers A \& Albarran JW (2007) A European survey of enteral nutrition practices and procedures in adults in intensive care units. J Clin Nurs 16, 2132-2141.

54. Thorensen L, Rothenberg E, Beck AM \& Irtun O (2008) The Scandinavian Nutrition Group Doctors and nurses on wards with greater access to clinical dieticians have better focus on clinical nutrition. J Hum Nutr Diet 21, 239-247.

55. Faisy C, Rabbat A, Kouchakji B \& Laaban JP (2000) Bioelectrical impedance analysis in estimating nutritional status and outcome of patients with chronic obstructive pulmonary disease and acute respiratory failure. Intensive Care Med 26, 518-525. 